\section{ORIGINAL RESEARCH}

L. Saba

R. Montisci

E. Raz

R. Sanfilippo

J.S. Suri

M. Piga

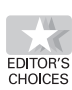

\title{
Association between Carotid Artery Plaque Type and Cerebral Microbleeds
}

BACKGROUND AND PURPOSE: CMBs have become increasingly recognized with the widespread use of MR imaging techniques that are sensitive to iron deposits. The purpose of this study was to correlate the presence of CMBs and carotid plaque characteristics.

MATERIAL AND METHODS: Seventy consecutive patients (47 men; 23 women; mean age, 65 years) were prospectively analyzed. Carotid arteries were studied using a 16-detector row CT scanner, whereas the brain was explored with an MR imaging 1.5T system. CMBs were studied using a T2*-weighted GRE sequence. CMBs were classified by an ordinal scale and carotid plaques were characterized based on their composition as fatty, mixed, or calcified. Patients were classified as symptomatic and asymptomatic. Chi-square and multiple logistic regression analyses, as well as ROCs, were calculated.

RESULTS: The prevalence of CMBs was $30 \%$. A statistically significant difference in CMB prevalence was observed between symptomatic $(46 \%)$ and asymptomatic $(19 \%)$ patients ( $P$ value $=.0021 ; \mathrm{OR}=$ 3.7). Correlation analysis demonstrated an association between the number of CMBs and the symptoms $(P=.0001)$. A statistically significant association was observed between the presence of fatty plaque and $\mathrm{CMBs}(P=.0019)$.

CONCLUSIONS: The results of this study suggest an association between the presence of carotid artery fatty plaque, symptoms, and CMBs. Moreover, we found that the presence (and entity) of CMBs may represent an indicator of cerebrovascular symptom severity.

ABBREVIATIONS: $\mathrm{AUC}=$ area under the $\mathrm{ROC}$ curve; $\mathrm{CMB}=$ cerebral microbleed; $\mathrm{GRE}=$ gradient recalled-echo; $\mathrm{HU}=$ Hounsfield unit; $\mathrm{MDCTA}=$ multidetector row $\mathrm{CT}$ angiography; $\mathrm{OR}=$ odds ratio; $\mathrm{ROC}=$ receiving operator characteristic

C MBs are small, rounded hypointensities visualized on GRE T2*-weighted MR imaging brain scans that are increasingly detected in patients with ischemic stroke. ${ }^{1-3}$ It has been suggested that microbleeds are a useful biomarker for pathologic damage to small vessels from hypertension or cerebral amyloid angiopathy, but little is known about the development of CMBs over time in patients with ischemic stroke, though they may have cumulative effects on brain function, including cognition. ${ }^{4}$ There are controversial results suggesting that the presence of cerebral microbleeds indicates an increased risk of bleeding complications from thrombolytic treatment or the use of antiplatelet drugs, ${ }^{5,6}$ and some authors found that presence of CMBs is related to risk of hemorrhagic transformation after ischemic stroke and to recurrence of spontaneous intracerebral bleeding. ${ }^{7,8}$

The question of whether CMBs are an expression of diffuse disease or the consequence of other factors, such as carotid plaque composition, is still debated, ${ }^{9}$ and the hypothesis we explore in this study is whether there might be a relationship between the presence of CMBs and the presence and characteristics of carotid plaque. Within the last several years, it was demonstrated that the composition of the carotid plaque plays

Received October 4, 2011; accepted after revision February 27, 2012

From the Departments of Radiology (L.S.), Vascular Surgery (R.M., R.S.), and Nuclear Medicine (M.P.), Azienda Ospedaliero Universitaria, di Cagliari, Cagliari, Italy; Department of Radiology (E.R.), New York University Langone Medical Center, New York, New York; Biomedical Engineering (J.S.S.), Idaho State University, Pocatello, Idaho; and Global Biomedical Technologies Inc. (J.S.S.), Roseville, California.

Please address correspondence to Luca Saba, MD, Department of Radiology, Azienda Ospedaliero Universitaria, di Cagliari - Polo di Monserrato s.s. 554 Monserrato, Cagliari, 09045, Italy; e-mail: lucasaba@tiscali.it

http://dx.doi.org/10.3174/ajnr.A3133 a fundamental role in stroke risk assessment, ${ }^{10-15}$ and MDCTA demonstrated to be an excellent and reproducible technique for the study of carotid artery plaque composition. ${ }^{16-21}$ We will test our hypothesis by studying carotid plaque type using MDCTA and the presence of CMBs using MR imaging.

\section{Materials and Methods}

\section{Study Design and Patient Population}

Institutional review board approval for this study was obtained. Based on a power calculation (type I error, $\alpha=0.05$; type II error, $\beta=0.1$; AUC null hypothesis value $=0.5$; AUC significant value $=0.75$, pooled group), we estimated that a sample size of at least 70 carotid arteries would be sufficient for investigating the relationship between carotid artery plaque type and CMBs. Therefore, 70 consecutive patients ( 47 men, 23 women; mean age, 65 years; range, $42-86$ years) were prospectively enrolled in this study from May 2009 to June 2010. All patients underwent MDCTA of supra-aortic vessels and brain MR imaging.

At our institute (Azienda Ospedaliera Universitaria di Cagliari), inclusion criteria for performing MDCTA of carotid arteries were previously published ${ }^{22,23}$ and are as follows: presence of a sonography examination that showed a pathologic stenosis $(50 \%$ of stenosis according to the NASCET criteria ${ }^{22-24}$ ) and/or a plaque alteration that was considered as the presence of a heterogeneous plaque, an irregular surface, intraplaque hemorrhage, and/or the presence of ulceration in the plaque. Another criterion for performing MDCTA was identified when the ultrasound could not provide adequate information about the degree of stenosis and plaque type. Known allergies to iodinated contrast material or elevated renal function tests were considered as exclusion criteria for the MDCTA examination. 


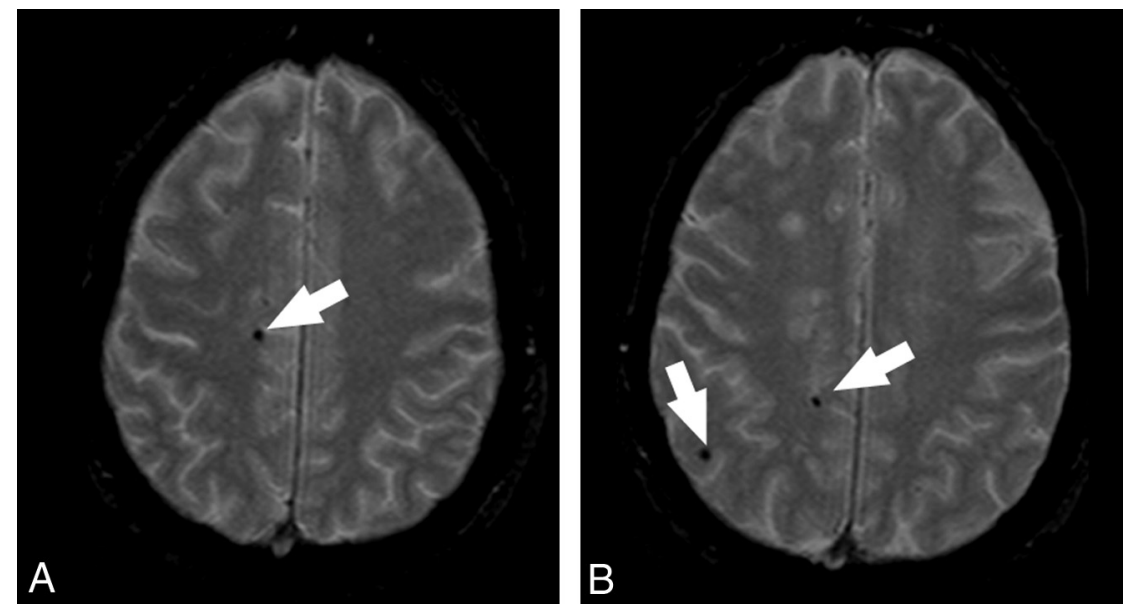

Fig 1. MR axial images that demonstrate the presence of CMBs. The white arrows indicate the CMBs.

We did not include those patients with a potentially confounding condition (ie, suspected embolism from a cardiac source, follow-up after carotid endarterectomy, intracerebral aneurysms, brain tumors) or with posterior cerebrovascular symptoms in this study.

At our institution, carotid arteries of asymptomatic patients were also studied in those patients who underwent cardiac interventions for coronary artery disease, aortic interventions, and lower-leg artery surgery, and in diabetics who were $>50$ years old.

\section{Classification of Cerebrovascular Symptoms}

Vascular risk factors and coexisting comorbidities and treatment known before stroke or transient ischemic attack are systematically recorded in our institution. Causes of stroke were classified according to Trial of Org 10172 in Acute Stroke Treatment criteria. ${ }^{25}$ We classified patients as symptomatic or asymptomatic according to the neurologic assessment documented in the clinical chart review. In the case of a TIA or stroke, a patient was considered symptomatic. TIA was regarded as an episode of neurologic dysfunction (hemiparesis, hemiparesthesia, dysarthria, dysphasia, or monocular blindness) not exceeding 24 hours. If the episode of neurologic dysfunction exceeded 24 hours, it was classified as a stroke. The time window to be included in the symptomatic group was 3 months; when a patient underwent MDCTA examination $>3$ months after the TIA or stroke event, he or she was excluded from the symptomatic group and included in the asymptomatic group. Diabetes was indicated by an abnormal fasting plasma glucose level $(>7.9 \mathrm{mmol} / \mathrm{l})$ or the current use of insulin or an oral hypoglycemic agent. Dyslipidemia was defined as abnormal fasting plasma cholesterol (low-attenuation lipoprotein cholesterol) levels (fasting cholesterol $>5.0 \mathrm{mmol} / \mathrm{l}$ ) or the current use of lipid-lowering agents (only if these agents were prescribed for a history of hyperlipidemia or dyslipidemia). Essential hypertension included those patients with systolic blood pressure $>140 \mathrm{~mm} \mathrm{Hg}$ and/or diastolic blood pressure $>90 \mathrm{~mm} \mathrm{Hg}$, or who were being treated with blood-pressure-lowering drugs. Cigarette smoking status was categorized as never/former (24 months) or current.

\section{MDCTA Technique}

All patients underwent MDCTA of the supra-aortic vessels with a 16 multidetector row CT system (Brilliance; Philips, Best, the Netherlands) using a technique previously described. ${ }^{24,26}$ Angiographic phase was obtained by injecting $80 \mathrm{~mL}$ of contrast medium (Ultravist 370; Bayer, Leverkusen, Germany) at a flow rate of $5 \mathrm{~mL} / \mathrm{s}$. A bolus tracking technique was used to calculate the correct timing of the scan. CT technical parameters included matrix $512 \times 512$; FOV 14-19 cm; mAs 180-220; kV 120-140; an intermediate reconstruction algorithm (C-filter) was used. Angiographic acquisition included the carotid siphon. None of the patients included in the study had a medical history of cardiac output failure or any contraindications to iodinated contrast media.

\section{Brain MR Technique}

Imaging examinations were performed with a Gyroscan 1.5T superconducting magnet (Philips) with a head coil, according to a standardized protocol. In all participants, conventional 2D T2*-weighted GRE MR imaging was performed (TR shortest; TE $23 \mathrm{~ms}$; flip angle $15^{\circ}$; in-plane resolution $0.9 \times 0.9 \mathrm{~mm}$; FOV $230 \mathrm{~mm}$; matrix $512 \times$ 512; section thickness $5 \mathrm{~mm}$; $0.5 \mathrm{~mm}$ intersection gap). Two-dimensional $\mathrm{T}^{\star}$-weighted GRE was performed with flow compensation, with an echo time of $20 \mathrm{msec}$ and a section thickness of $5 \mathrm{~mm}$, in line with conventional settings. ${ }^{27-29}$ DWI was performed with single-shot spin-echo with 2 diffusion sensitivity values of 0 and 1000 seconds/ $\mathrm{mm}^{2}$ along the transverse axis. In addition to $\mathrm{T} 2^{*}$-weighted GRE and DWI sequences, axial spin-echo T1-weighted images (500-600/15/2 for TR/TE/excitations) and fast spin-echo T2-weighted images (2200-3200/80-120/1,2 for TR/TE/excitations; turbo factor, 24) were also obtained with the same section thickness.

\section{CMBs Definition and Rating}

Acquisition date and participant identification were removed from all images. Two experienced radiologists (L.S. and E.R., with 10 and 8 years of experience, respectively), unaware of the clinical data, reviewed, blinded to each other, all images for presence and location of CMBs. This analysis was performed to evaluate the interobserver variability. After a 3-week gap, datasets were reanalyzed in consensus to obtain the reference parameter for the statistical analysis. At this time, the T1-weighted images were additionally used to confirm the location of microbleeds, as the inherent properties of microbleeds will cause these to appear as a focus of low signal intensity (without marked blooming) on the T1-weighted image as well. Thus, the T1weighted image further facilitated differentiation of microbleeds from calcification in the ventricle or from sulcal vessels. ${ }^{30}$ The presence, location, and number of all cerebral microbleeds was recorded. In our study, CMBs were considered as focal, rounded, or circular areas of very low signal intensity that were smaller than $10 \mathrm{~mm}$ in size, 

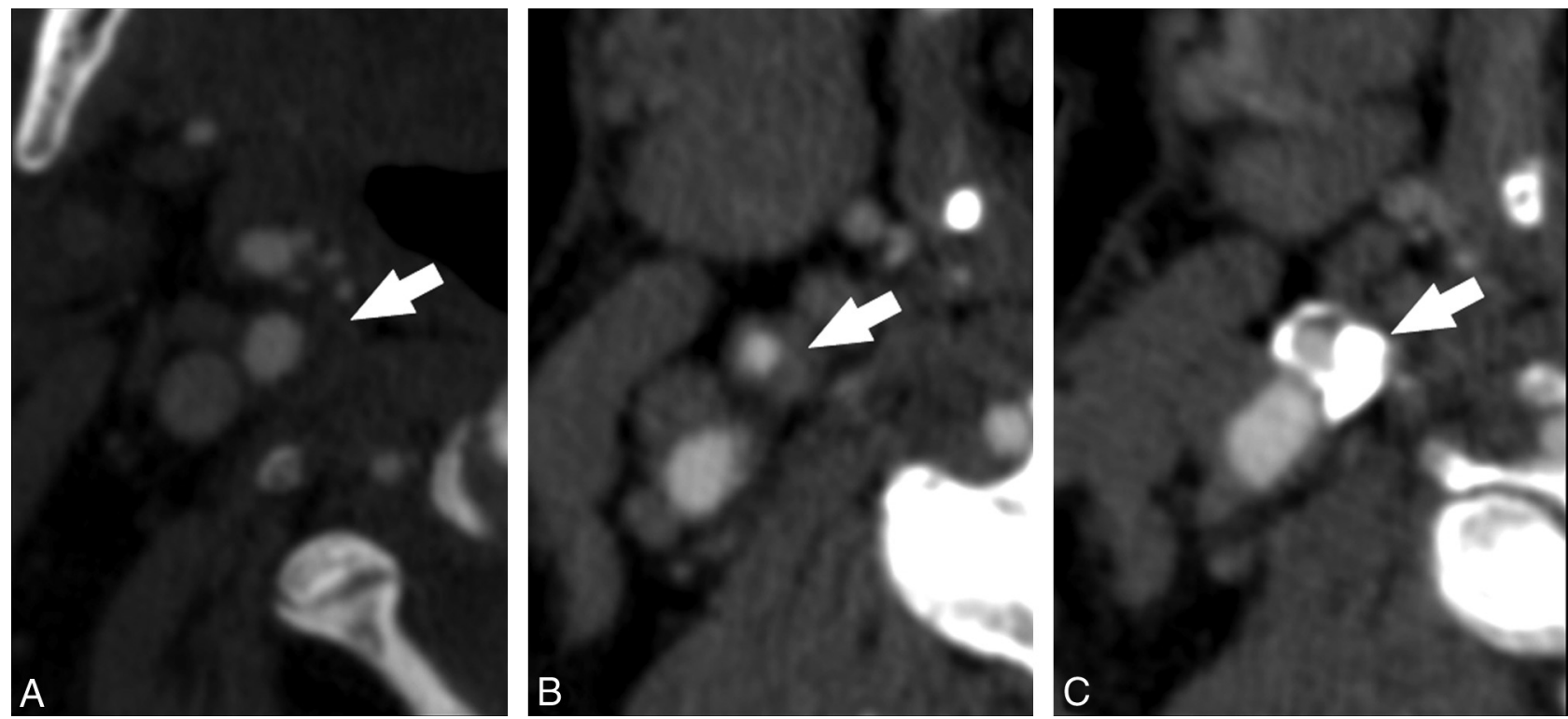

Fig 2. CTA axial images that demonstrate $(A)$ fatty plaque, $(B)$ mixed plaque, and $(C)$ calcified plaque.

as suggested by Jeerakathil et $\mathrm{al}^{31}$ (Fig 1). In accordance with consensus guidelines on standards for neuroimaging in vascular cognitive impairment, we considered $2 \mathrm{~mm}$ as the lower size limit. ${ }^{32} \mathrm{We}$ also categorized the locations of the $\mathrm{CMBs}^{27,31}$ as lobar (cortical gray matter and subcortical white matter), deep (deep gray matter [basal ganglia and thalamus] and deep white matter [corpus callosum]), or infratentorial (brain stem and cerebellum). Symmetric hypointensities in the globi pallidi, likely to represent calcification, and sulcal flow voids from cortical vessels were disregarded. ${ }^{33}$

Microbleeds were classified in each cerebral hemisphere (and for infratentorial lesions, these were considered in the right/left cerebellum of right/left side of the brain stem) as absent (grade 1), mild (grade 2; total number of microbleeds, 1-2), moderate (grade 3; total number of microbleeds, 3-10), and severe (grade 4; total number of microbleeds, $>10$ ), according to Lee and colleagues. ${ }^{34}$

\section{Carotid Artery Plaque Classification}

A single experienced radiologist (L.S., 10 years of experience), blinded to the clinical information and MR brain analysis, performed all MDCTA measurements. MDCTA axial images were analyzed, with a varying magnification from $120 \%-400 \%$, in comparison with the acquisition. Window parameters (width and level) were not preset. ${ }^{18,35}$ We categorized different types of plaques, dividing them into fatty plaques, with attenuation $<60 \mathrm{HU}$; mixed plaques, with attenuation between 60-120 HU; and calcified plaques, with attenuation $>130 \mathrm{HU}$ (Fig 2). To measure HU value, a circular or elliptical ROI cursor in the predominant area of plaque was used by the method outlined according to Saba et al. ${ }^{12}$ Regions of beam hardening in calcified areas were also excluded. The radiologist took the measurements at the point of major stenosis, which was identified by elaborating oblique axial images normal to the ICA lumen centerline.

\section{Statistical Analysis}

For the analysis of the data, we considered the carotid and cerebral hemisphere of each side as an independent unit. Therefore, the total number of studied carotid arteries and brain hemispheres was 140. In symptomatic patients, we considered the ipsilateral carotid artery and brain hemisphere as "symptomatic," and the contralateral carotid
Table 1: Patient characteristics

\begin{tabular}{lccc}
\hline & Presence of CMBs & Absence of CMBs & $P$ Value \\
\hline Age (mean [SD]) & 63 years (14.5 years) & 65 years (13.3 years) & .42 \\
Sex (male) & $71 \%$ & $67 \%$ & .74 \\
Tobacco user & $52 \%$ & $37 \%$ & .22 \\
Hypertension & $62 \%$ & $45 \%$ & .19 \\
CAD & $52 \%$ & $53 \%$ & .95 \\
Diabetes & $21 \%$ & $12 \%$ & .36 \\
Dyslipidemia & $43 \%$ & $49 \%$ & .64 \\
Statins and other & $43 \%$ & $33 \%$ & .41 \\
$\quad$ lipid-lowering & & & \\
$\quad$ drugs & & &
\end{tabular}

Note:-CAD indicates coronary artery disease.

artery and brain hemisphere as "asymptomatic," whereas in asymptomatic patients, both carotids and brain hemispheres were considered "asymptomatic." In symptomatic patients, we considered a symptomatic side and asymptomatic side according to the clinical symptoms, whereas in asymptomatic patients, both sides were considered asymptomatic. We tested the interobserver reliability for $\mathrm{CMB}$ rating using the Cohen $\kappa$ test. ${ }^{36}$ Continuous data were described as the mean value $\pm \mathrm{SD}$ and were compared using the Wilcoxon signed rank test. ROC curve analysis was performed to identify thresholds in number of CMBs associated with presence of symptoms. The Student $t$ test for paired samples was used to test the difference in the number of CMBs in the same subjects between the side with the fatty plaques and the side without the fatty plaques. Presence or absence of cerebrovascular symptoms was compared between patients with and without $\mathrm{CMBs}$, according to the different plaque types, using a Cochran-Mantel-Haenszel test. Logistic regression and stepwise logistic regression analysis was used to identify the most accurate linear combinations of distinguishing variables. R software (http://www.r-project.org) was used for statistical analyses.

\section{Results}

The clinical characteristics of the studied patients are shown in Table 1. We observed 39 patients (55.7\%) with ischemic symptoms (12 with strokes, 20 with cerebral TIAs, 7 with monocular TIAs) and 31 without ischemic symptoms. There- 


\begin{tabular}{lccccc}
\hline Table 2: Plaque type analysis & & \\
\hline & $\begin{array}{c}\text { Symptomatic } \\
\text { Patient CMBs } \\
(n=39)\end{array}$ & & \multicolumn{2}{c}{$\begin{array}{c}\text { Asymptomatic } \\
\text { Patient CMBs } \\
(n=87)\end{array}$} \\
\cline { 2 - 3 } \cline { 5 - 6 } & Presence & Absence & & Presence & Absence \\
\hline $\begin{array}{c}\text { Fatty plaques } \\
(n=37)\end{array}$ & 13 & 9 & & 7 & 8 \\
$\begin{array}{c}\text { Mixed plaques } \\
(n=49)\end{array}$ & 6 & 7 & & 3 & 33 \\
$\begin{array}{c}\text { Calcified plaques } \\
(n=40)\end{array}$ & 1 & 3 & & 1 & 35 \\
\hline
\end{tabular}

fore, we had 39 symptomatic sides and 101 asymptomatic sides. The prevalence of the CMBs was 30\% (21/70); in 16 patients, we found CMBs in both hemispheres, and in 5 patients, CMBs were found in only 1 hemisphere; thus, we had 37 hemispheres with CMBs and 103 hemispheres without CMBs.

On the symptomatic side, there was a 46\% (18/39) prevalence of CMBs, whereas on the asymptomatic side, there was a 19\% (19/101) prevalence; a statistically significant difference between CMB presence was observed, with a $P$ value of .0021 and an odds ratio of 3.7.

The radiologists showed very good interobserver agreement in the identification of CMBs, with a $\kappa$ value of 0.81 .

\section{Relationship of Cerebrovascular Symptoms and CMBs}

We performed an analysis to evaluate the relationship between the number of CMBs (and their grade, from 1 to 4 ) and the presence of cerebrovascular symptoms. We observed a statistically significant correlation between the presence of symptoms and the number of CMB (correlation coefficient = $0.319 ; P$ value $=.0001$ ) and the $\mathrm{CMBs}$ grade (correlation coefficient $=0.314 ; P$ value $=.0001)$. We also analyzed the association between the different subtypes of cerebrovascular symptoms (stroke, TIA, and amaurosis) with the presence of CMBs, and found a strong association between stroke $(P=$ $.0001 ;$ OR $=10.71)$, but no association with TIA $(P=.347$; $\mathrm{OR}=1.62)$ and amaurosis $(P=.895 ; \mathrm{OR}=1.12)$.

\section{Relationship of Plaque Type and CMBs}

In the plaque type analysis of the 140 carotid arteries, we excluded 14 because there was no evidence of plaque. In the remaining 126 carotid arteries, we found 37 fatty plaques (29.4\%), 49 mixed plaques (38.9\%), and 40 calcified plaques $(31.8 \%)$. In 2 cases CMBs were found in the 14 excluded hemispheres, supplied by an regular ICA, and therefore the plaque type analysis was performed by considering 35 hemispheres with CMBs. All 39 symptomatic hemispheres had a plaque in the carotid artery and therefore were included in the analysis. A summary of the plaque type analysis, according to the presence of CMBs and symptoms, is given in Table 2. The Cochran-Mantel-Haenszel test showed that there was a statistically significant increase $(P=.012$; OR $=3.465)$ in $\mathrm{CMB}$ prevalence in symptomatic patients versus that in asymptomatic patients.

In analyzing the relationship between CMBs and the type of plaque, we performed a correlation analysis and found a statistically significant correlation with the fatty plaques (correlation coefficient $=0.271 ; P$ value $=.0019)$, whereas an inverse statistical trend was observed between CMBs and cal- cified plaques (correlation coefficient $=-0.152 ; P$ value $=$ .0851). No statistically significant association was detected between CMBs and mixed plaque type (correlation coefficient $=$ $-0.117 ; P$ value $=.188)$.

In analyzing whether $\mathrm{CMB}$ are an expression of diffuse disease or the consequence of an unstable plaque, we performed a Student $t$ test for paired samples and observed a statistical trend ( $P$ value of .0699).

\section{ROC Curve Analysis}

The ROC curve analysis for symptoms versus number of CMBs is shown in Fig $3 A$. The ROC area under the curve $(\mathrm{Az})$ for the direct-mm method was 0.646 (95\% CI 0.561-0.724; $\mathrm{SD}=0.054 ; P=.0069)$. Using the ROC analysis, we calculated the sensitivity, specificity, positive likelihood ratio and negative likelihood ratio values for the direct-mm method; a summary of these values is provided in Table 3. By analyzing threshold values, we observed that the presence of $5 \mathrm{CMBs}$ are associated with a specificity of $90.2 \%$ for the presence of cerebrovascular symptoms. We also performed the ROC curve analysis for stroke, TIA, and amaurosis (Fig 3B-D) and we obtained the following AUCs, respectively: $0.802(P=.0001)$, $0.464(P=.61)$, and $0.526(P=.819)$, by confirming that there is an association between CMBs and stroke but not between CMBs and TIA or amaurosis

\section{Logistic Regression Analysis}

To exclude any confounding effect between CMBs and other potentially related variables, logistic regression analysis was performed with $\mathrm{CMB}$ as the dependent variable, plaque type as the independent variable, and other factors such as hypertension, diabetes, tobacco use, coronary artery disease, and dyslipidemia as cofactors (Table 4). This analysis demonstrated that the only statististically significant association was present between CMBs and fatty plaques. Next, the stepwise logistic regression analysis was performed; the results are provided in Table 5. In the stepwise logistic regression model, the dependent variable was the presence of cerebrovascular symptoms, and we included the following parameters: sex of the patient, coronary artery disease, diabetes, hypertension, dyslipidemia, tobacco use, type of plaque (fatty, mixed, calcified), and presence of CMBs. The stepwise logistic regression model indicated that CMBs and fatty plaques were both independent variables associated with cerebrovascular symptoms ( $P$ values, respectively, .0026 and .0018). Other variables did not demonstrate a significant statistical association with cerebrovascular symptoms.

\section{Results Based on CMB Location}

In 13 cases (9.3\%), we had only lobar CMBs; in 14 cases $(10 \%)$, we found only deep CMBs; whereas in 9 cases $(8.2 \%)$, we found combined (both deep and lobar) CMBs. We observed CMBs in the cerebellum (right side) in only 1 case $(0.7 \%)$.

\section{Discussion}

In the past, it has been suggested that CMBs may be a biomarker for pathologic damage to small vessels from hypertension or cerebral amyloid angiopathy, ${ }^{37,38}$ but some important areas of clinical uncertainty regarding microbleeds remain, in particular, their potential role in predicting the risk of intra- 

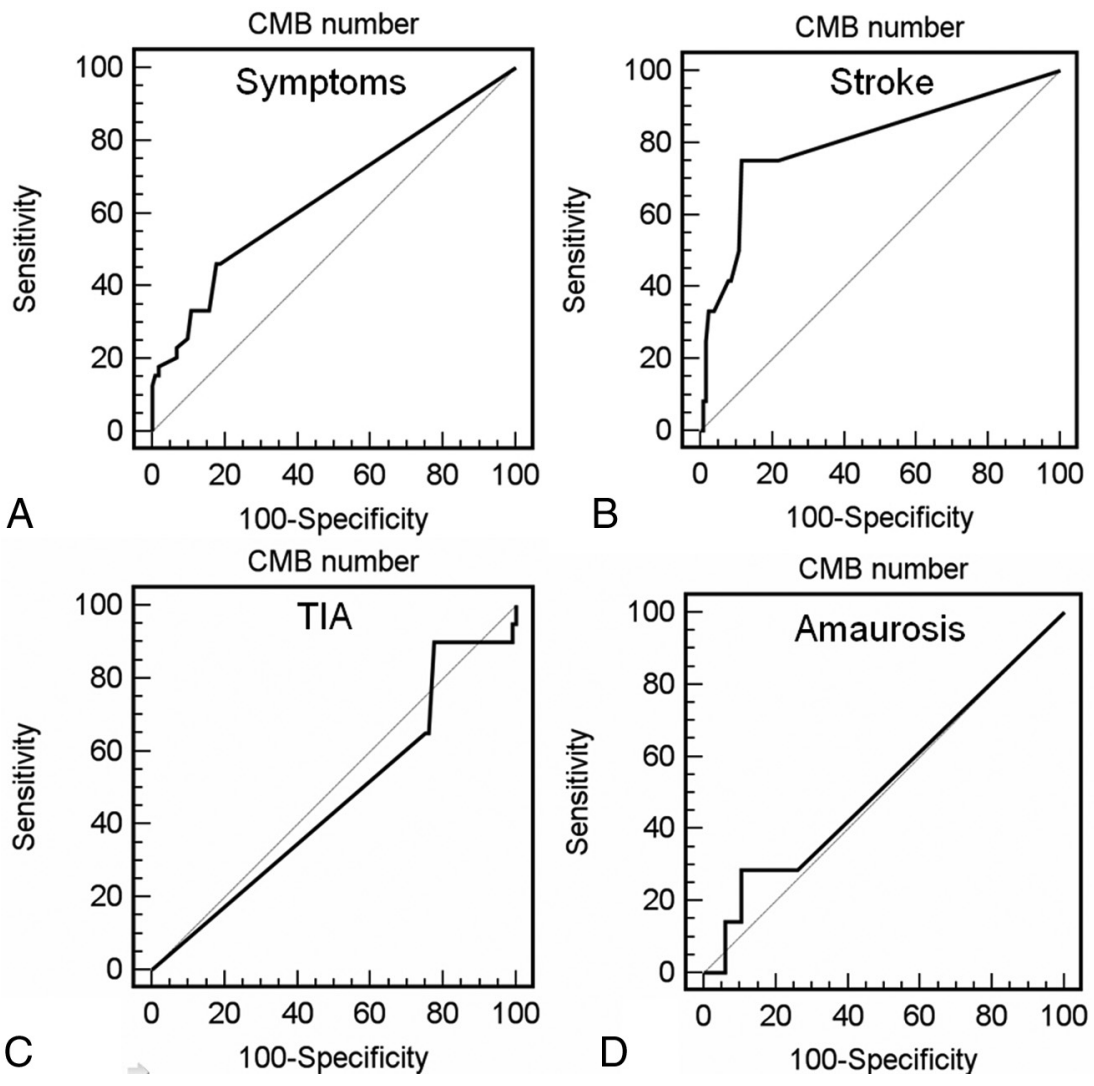

Fig 3. ROC curves between presence of CMBs and $(A)$ cerebrovascular symptoms, $(B)$ stroke, $(C)$ TIA, and $(D)$ amaurosis.

\begin{tabular}{lcccccc}
\hline \multicolumn{7}{l}{ Table 3: ROC curve analysis } \\
\hline Criterion $^{\mathrm{a}}$ & Sensitivity & \multicolumn{10}{c}{$95 \% \mathrm{Cl}$} & Specificity & $95 \% \mathrm{Cl}$ & $+\mathrm{LR}$ & $-\mathrm{LR}$ \\
\hline$\geq 0$ & 100.00 & $90.9-100.0$ & 0.00 & $0.0-3.6$ & 1.00 & \\
$>0$ & 46.15 & $30.1-62.8$ & 81.37 & $72.4-88.4$ & 2.48 & 0.66 \\
$>1$ & 46.15 & $30.1-62.8$ & 82.35 & $73.5-89.2$ & 2.62 & 0.65 \\
$>2$ & 33.33 & $19.1-50.2$ & 84.31 & $75.8-90.8$ & 2.12 & 0.79 \\
$>4$ & 33.33 & $19.1-50.2$ & 89.22 & $81.5-94.5$ & 3.09 & 0.75 \\
$>5$ & 25.64 & $13.1-42.1$ & 90.20 & $82.7-95.2$ & 2.62 & 0.82 \\
$>6$ & 23.08 & $11.2-39.3$ & 93.14 & $86.4-97.2$ & 3.36 & 0.83 \\
$>7$ & 20.51 & $9.3-36.5$ & 93.14 & $86.4-97.2$ & 2.99 & 0.85 \\
$>8$ & 17.95 & $7.6-33.5$ & 98.04 & $93.1-99.7$ & 9.15 & 0.84 \\
$>9$ & 15.38 & $5.9-30.5$ & 98.04 & $93.1-99.7$ & 7.85 & 0.86 \\
$>11$ & 15.38 & $5.9-30.5$ & 99.02 & $94.6-99.8$ & 15.69 & 0.85 \\
$>15$ & 12.82 & $4.3-27.4$ & 100.00 & $96.4-100.0$ & & 0.87 \\
$>20$ & 0.00 & $0.0-9.1$ & 100.00 & $96.4-100.0$ & & 1.00 \\
\hline
\end{tabular}

Note:- $\mathrm{Cl}$ indicates confidence interval; LR, likelihood ratio

${ }^{a}$ Number of cerebral microbleeds.

cranial hemorrhage (especially in patients treated with thrombolytics or antithrombotics), their contribution to cognitive impairment, and their value for diagnosing small vessel diseases. $^{4,39}$

In our population, the prevalence of the CMBs was 30\% $(21 / 70)$; this result is a little higher compared with the prevalence of CMBs detected in the recently published Rotterdam scan study ${ }^{40}(28 \%)$; however, in our research, the percentage of symptomatic patients is higher compared with the Rotterdam study and this fact may justify the higher value. The prevalence of CMBs is lower compared with Vernooij et al, ${ }^{30}$ where it was $35.5 \%$; however, this difference can be explained with the different technique used by these authors $\left(3 \mathrm{D} \mathrm{T}^{*}\right.$ weighted GRE).
We observed a statistically significant difference in $\mathrm{CMB}$ prevalence in the symptomatic versus asymptomatic side $(P$ value $=.0021 ; \mathrm{OR}=3.7)$. This result may suggest that the $\mathrm{CMBs}$ are associated with the presence of cerebrovascular events and are confirmed by the ROC curve analysis (AUC = $0.646 ; P$ value $=0069)$ and by the logistic regression analysis $(P=.0026)$. However, in the analysis of our results, it is important to underline that in the symptomatic hemispheres, the prevalence of fatty plaques was markedly higher (56\%) compared with the mixed (33\%) and calcified plaques (10\%). This fact may represent a bias and, therefore, this association should be confirmed in further longitudinal and prospective analyses. However, the logistic regression analysis showed that both CMBs and fatty plaques are independent variables associated with the presence of cerebrovascular symptoms.

We also analyzed the relationship between the different type of cerebrovascular symptoms (stroke, TIA, amaurosis) and observed unexpected results: there was a strong association between the stroke and presence of CMBs $(P=.0001$; OR $10.71)$ but no association with TIA $(P=.347$; OR 1.62) and amaurosis $(P=.895$; OR $=1.12)$. Moreover, the ROC curve analysis (Fig $3 B$ ) demonstrated that the number of CMBs has a statistically significant association with stroke (AUC = $0.802 ; P=.0001)$. This is an interesting finding-is the presence of CMBs an indicator of the cerebrovascular symptom severity (stroke $>$ TIA $>$ amaurosis)? Or is it the severity of the cerebrovascular event that may determine the presence (or not) of $\mathrm{CMB}$ and its entity? These data are concordant with previous research by Ovbiagele et $\mathrm{al},{ }^{41}$ where the authors demonstrated that CMBs are more frequently noted in hospital- 


\begin{tabular}{lccccc}
\hline \multicolumn{2}{l}{ Table 4: Logistic regression model: CMB dependent variable } & & & \\
\hline Variable & Coefficient & SE & $P$ & Odds Ratio & \\
\hline Age & 0.0227 & 0.0207 & 0.2721 & 1.023 & $0.95 \%$ CI \\
CAD & 0.6966 & 0.4579 & 0.1282 & 2.007 & 0.8823 to 1.0653 \\
Diabetes & 0.8106 & 1.218 & 0.5057 & 0.4446 & 0.0409 to 4.9239 \\
Hypertension & 0.0461 & 0.5864 & 1.0471 & 0.3318 to 3.3047 \\
Dyslipidemia & 0.096 & 0.4907 & 0.9374 & 1.1008 & 0.4207 to 2.8801 \\
Gender & 1.0567 & 0.7076 & 0.8449 & 2.877 & 0.7189 to 11.5139 \\
Tobacco use & 0.4274 & 0.5807 & 0.45618 & 1.5332 & 0.4913 to 4.7850 \\
Calcified plaques & 0.0316 & 0.5843 & 0.9568 & 1.0321 & 0.3284 to 3.2443 \\
Mixed plaques & 0.7566 & 0.678 & 0.2645 & 2.131 & 0.5642 to 8.0490 \\
Fatty plaques & 1.5647 & 0.4499 & 0.0005 & 4.7811 & 1.9796 to 11.5471 \\
\hline
\end{tabular}

Note:- $\mathrm{Cl}$ indicates confidence interval; SE, standard error; $\mathrm{CAD}$ indicates coronary artery disease.

Table 5: Stepwise logistic regression model

\begin{tabular}{lccccc}
\hline Variable & Coefficient & SE & $P$ & Odds Ratio & \multicolumn{1}{c}{$95 \% \mathrm{Cl}$} \\
\hline CMBs & 1.3977 & 0.464 & 0.0026 & 4.046 & 1.6296 to 10.0453 \\
Fatty plaques & 1.4005 & 0.4481 & 0.0018 & 4.0572 & 1.6859 to 9.7637 \\
Constant & -1.8148 & & & &
\end{tabular}

Note:-Dependent variable is presence/absence of cerebrovascular symptoms. $\mathrm{Cl}$ indicates confidence interval; SE, standard error.

ized stroke. It is our opinion that the presence of CMBs may represent an indicator for cerebrovascular symptom severity ( stroke $>$ TIA > amaurosis); however, to correctly verify this hypothesis, a specific prospectively designed study should be performed.

By analyzing the relationship between CMBs and the type of the plaque, we found a statistically significant correlation with the fatty plaques (correlation coefficient $=0.271 ; P$ value $=.0019$ ), whereas an inverse statistical trend was observed between CMBs and calcified plaques (correlation coefficient $=-0.152 ; P$ value $=.0851)$. No statistically significant association was detected between $\mathrm{CMBs}$ and mixed plaque type (correlation coefficient $=-0.117 ; P$ value $=.188$ ).

By comparing the hemispheres of the same patient, concerning the number of microbleeds, we also tried to explore the question of whether CMBs are an expression of a diffuse disease or the consequence of an unstable plaque. In the 16 patients with CMBs with a carotid fatty plaque, and with the contralateral carotid artery mixed or calcified, we observed that there was a statistical trend $(P=.0699)$ toward the increase of CMBs in those hemispheres ipsilateral to the presence of a carotid fatty plaque. However, for the purpose of this analysis, 16 patients represent a very small population and we think that this kind of hypothesis should be tested using bigger samples. Moreover, our results should be further tested and confirmed, as we explored abnormalities that are from different vascular territories: 1 in the microvascular bed and the other on a large vessel.

In our study, we observed that the distribution of CMBs is quite homogeneous and that $8.2 \%$ of patients had both deep and lobar CMBs. Our results are concordant with previous research by Staals et al, ${ }^{33}$ where $8.9 \%$ of patients had only lobar CMBs, $13.8 \%$ had only deep CMBs, and 5.7\% had combined (both deep and lobar) CMBs. In our population, we did not find any CMBs in the brain stem. In the article by Gregoire et $\mathrm{al},{ }^{42}$ the CMBs were most prevalent in the lobar region $(17 \%-$ $22 \%)$, followed by the deep (14\%-15\%) and infratentorial regions $(6 \%-12 \%)$.

There are some limitations of our research. First, the pa- tient population included in our data base had significant symptomatic cerebral vascular diseases $(55.7 \%)$; the prevalence of disease in this study was higher compared with the general population, and this fact may relate to differences in the pool of the plaque composition. Second, it is possible that, in some cases, there is a potential misclassification of CMBs. In fact, other structures in the brain may resemble microbleeds on MR images, such as calcifications in basal ganglia or the pineal gland. However, we believe that we did not misclassify other structures as CMBs, as calcifications in the brain usually have a typical location and shape, and when located in the basal ganglia, they are usually symmetric in distribution.

\section{Conclusions}

The results of this study suggest an increased number of CMBs in symptomatic patients and indicate an association between the presence of carotid artery fatty plaque, symptoms, and CMBs. Our results seems to indicate that the presence (and entity) of CMBs may represent an indicator of cerebrovascular symptom severity.

\section{References}

1. Werring DJ, Coward LJ, Losseff NA, et al. Cerebral microbleeds are common in ischemic stroke but rare in TIA. Neurology 2005;65:1914-18

2. Fazekas F, Kleinert R, Roob G, et al. Histopathologic analysis of foci of signal loss on gradient-echo $\mathrm{T} 2 *$-weighted MR images in patients with spontaneous intracerebral hemorrhage: evidence of microangiopathy-related microbleeds. AJNR Am J Neuroradiol 1999;20:637-42

3. Atlas SW, Mark AS, Grossman RI, et al. Intracranial hemorrhage: gradientecho MR imaging at $1.5 \mathrm{~T}$ : comparison with spin-echo imaging and clinical applications. Radiology 1988;168:803-07

4. Werring DJ, Frazer DW, Coward LJ, et al. Cognitive dysfunction in patients with cerebral microbleeds on T2*-weighted gradient-echo MRI. Brain 2004; 127:2265-75

5. Leblanc R, Haddad G, Robitaille Y. Cerebral hemorrhage from amyloid angiopathy and coronary thrombolysis. Neurosurgery 1992;31:586-90

6. Wong KS, Chan YL, Liu JY, et al. Asymptomatic microbleeds as a risk factor for aspirin-associated intracerebral hemorrhages. Neurology 2003;60:511-13

7. Naka H, Nomura E, Wakabayashi S, et al. Frequency of asymptomatic microbleeds on $\mathrm{T} 2{ }^{\star}$-weighted MR images of patients with recurrent stroke: association with combination of stroke subtypes and leukoaraiosis. AJNR Am J Neuroradiol 2004;25:714-19

8. Imaizumi T, Horita Y, Hashimoto Y, et al. Dotlike hemosiderin spots on T2*- 
weighted magnetic resonance imaging as a predictor of stroke recurrence: a prospective study. J Neurosurg 2004;101:915-20

9. Bos D, Ikram MA, Elias-Smale SE, et al. Calcification in major vessel beds relates to vascular brain disease. Arterioscler Thromb Vasc Biol 2011;31:2331-37

10. Naghavi M, Libby P, Falk E, et al. From vulnerable plaque to vulnerable patient: a call for new definitions and risk assessment strategies: Part II. Circulation 2003;108:1772-78

11. Wintermark M, Jawadi SS, Rapp JH, et al. High-resolution CT imaging of carotid artery atherosclerotic plaques. AJNR Am J Neuroradiol 2008;29:875-82

12. Saba L, Montisci R, Sanfilippo R, et al. Multidetector row CT of the brain and carotid artery: a correlative analysis. Clin Radiol 2009;64:767-78

13. Moody AR, Murphy RE, Morgan PS, et al. Characterization of complicated carotid plaque with magnetic resonance direct thrombus imaging in patients with cerebral ischemia. Circulation 2003;107:3047-52

14. Saba L. CT imaging in the carotid artery. In: Suri JS, Kathuria C, Molinari F, eds. Atherosclerosis Disease Management. New York: Springer-Verlag, 2010: 333-76

15. Rozie S, de Weert TT, de Monyé C, et al. Atherosclerotic plaque volume and composition in symptomatic carotid arteries assessed with multidetector CT angiography; relationship with severity of stenosis and cardiovascular risk factors. Eur Radiol 2009;19:2294-301

16. Saba L, Sanfilippo R, Pascalis L, et al. Carotid artery abnormalities and leukoaraiosis in elderly patients: evaluation with MDCT. AJR Am J Roentgenol 2009;192:W63-70

17. Saba L, Mallarini G. Fissured fibrous cap of vulnerable carotid plaques and symptomaticity: are they correlated? Preliminary results by using multi-detector-row CT angiography. Cerebrovasc Dis 2009;27:322-27

18. Saba L, Mallarini G. Window settings for the study of calcified carotid plaques with multidetector CT angiography. AJNR Am J Neuroradiol 2009;30:1445-50

19. Wintermark M, Arora S, Tong E, et al. Carotid plaque computed tomography imaging in stroke and nonstroke patients. Ann Neurol 2008;64:149-57

20. de Weert TT, Ouhlous M, Meijering E, et al. In vivo characterization and quantification of atherosclerotic carotid plaque components with multidetector computed tomography and histopathological correlation. Arterioscler Thromb Vasc Biol 2006;26:2366-72

21. de Weert TT, Ouhlous M, Zondervan PE, et al. In vitro characterization of atherosclerotic carotid plaque with multidetector computed tomography and histopathological correlation. Eur Radiol 2005;15:1906-14

22. Saba L, Pascalis L, Sanfilippo R, et al. Carotid artery wall thickness and leukoaraiosis: preliminary results using multidetector row $\mathrm{CT}$ angiography. AJNR Am J Neuroradiol 2011;32:955-61

23. Saba L, Sanfilippo R, Anzidei M, et al. Stenosis Asymmetry Index (SAI) between symptomatic and asymptomatic patients in the analysis of carotid arteries. A study using CT angiography. Eur J Radiol 2012;81:77-82

24. Saba L, Sanfilippo R, Montisci R, et al. Carotid artery wall thickness using CT: inter- and intraobserver agreement analysis. AJNR Am J Neuroradiol 2012 March 1 [Epub ahead of print]
25. Fure B, Wyller TB, Thommessen B. TOAST criteria applied in acute ischemic stroke. Acta Neurol Scand 2005;112:254-58

26. Saba L, Mallarini G. Carotid plaque enhancement and symptom correlations: an evaluation by using multidetector row CT angiography. AJNR Am J Neuroradiol 2011;32:1919-25

27. Roob G, Schmidt R, Kapeller P, et al. MRI evidence of past cerebral microbleeds in a healthy elderly population. Neurology 1999;52:991-94

28. Kinoshita T, Okudera T, Tamura H, et al. Assessment of lacunar hemorrhage associated with hypertensive stroke by echo-planar gradient-echo $\mathrm{T}^{*}$ weighted MRI. Stroke 2000;31:1646-50

29. Alemany M, Stenborg A, Terent A, et al. Coexistence of microhemorrhages and acute spontaneous brain hemorrhage: correlation with signs of microangiopathy and clinical data. Radiology 2006;238:240-47

30. Vernooij MW, Ikram MA, Wielopolski PA, et al. Cerebral microbleeds: accelerated 3D T2*-weighted GRE MR imaging versus conventional 2D T2* weighted GRE MR imaging for detection. Radiology 2008;248:272-77

31. Jeerakathil T, Wolf PA, Beiser A, et al. Cerebral microbleeds: prevalence and associations with cardiovascular risk factors in the Framingham Study. Stroke 2004;35:1831-35

32. Hachinski V, Iadecola C, Petersen RC, et al. National Institute of Neurological Disorders and Stroke-Canadian Stroke Network Vascular Cognitive Impairment Harmonization Standards. Stroke 2006;37:2220-41

33. Staals J, van Oostenbrugge RJ, Knottnerus IL, et al. Brain microbleeds relate to higher ambulatory blood pressure levels in first-ever lacunar stroke patients. Stroke 2009;40:3264-68

34. Lee $\mathrm{SH}, \mathrm{Bae} \mathrm{HJ}$, Yoon BW, et al. Low concentration of serum total cholesterol is associated with multifocal signal loss lesions on gradient-echo magnetic resonance imaging: analysis of risk factors for multifocal signal loss lesions. Stroke 2002;33:2845-49

35. Saba L, Mallarini G. MDCTA of carotid plaque degree of stenosis: evaluation of interobserver agreement. AJR Am J Roentgenol 2008;190:W41-46

36. Landis JR, Koch GG. The measurement of observer agreement for categorical data. Biometrics 1977;33:159-74

37. Vernooij MW, van der Lugt LA, Ikram MA, et al. Prevalence and risk factors of cerebral microbleeds: the Rotterdam Scan Study. Neurology 2008;70:1208-14

38. Pantoni L. Cerebral small vessel disease: from pathogenesis and clinical characteristics to therapeutic challenges. Lancet Neurol 2010;9:689-701

39. Cordonnier C, Al-Shahi SR, Wardlaw J. Spontaneous brain microbleeds: systematic review, subgroup analyses and standards for study design and reporting. Brain 2007;130:1988-2003

40. Poels MM, Ikram MA, van der Lugt A, et al. Incidence of cerebral microbleeds in the general population: the Rotterdam Scan Study. Stroke 2011;42:656-61

41. Ovbiagele B, Saver JL, Sanossian N, et al. Predictors of cerebral microbleeds in acute ischemic stroke and TIA patients. Cerebrovasc Dis 2006;22:378-83

42. Gregoire SM, Chaudhary UJ, Brown MM, et al. The Microbleed Anatomical Rating Scale (MARS): reliability of a tool to map brain microbleeds. Neurology 2009;73:1759-66 